\title{
Oral Staphylococcus in Older Subjects with Rheumatoid Arthritis
}

\author{
Jed J. Jacobson, DDS, 'Bonnie Patel, DDS, "Gillian Asher, BS," James O. Woolliscroft, MD, ${ }^{\dagger}$ and \\ Dennis Schaberg, $M D^{\ddagger}$
}

OBJECTIVE: To determine if Staphylococcus aureus and Staphylococcus epidermidis, etiologic bacterial agents to late prosthetic joint infections (LPJI), are more prevalent in the oral flora of older individuals with rheumatoid arthritis (RA) than in an age and gender-matched nonarthritic control population (NA).

DESIGN: Cultures were obtained from the nares, oropharynx, saliva, tongue, and gingival crevice, and the results were compared between older patients with RA and controls.

SETTING: University of Michigan Medical Center, Ann Arbor, VA Medical Center, and University of Michigan School of Dentistry.

PARTICIPANTS: A total of 111 community-dwelling subjects with a diagnosis of RA and 83 gender-matched control subjects.

MEASUREMENTS: Colistin nalidixic acid agar plates with $5 \%$ sheep's blood were inoculated and incubated. Isolates were speciated using the API Staph $\operatorname{Trac}^{\circledR}$ micro method and catalase and coagulase tests.

MAIN RESULTS: Individuals with RA had a higher prevalence of $S$. aureus isolated from the oral cavity. However, only the oropharynx and tongue revealed higher rates; all other sites were insignificant. The presence of oral $S$. aureus was associated with xerostomia. Staphylococcus epidermidis was not detected from any of the oral sites sampled. Sixtytwo percent (10/16) of the $S$. aureus isolates from the RA subjects were resistant to penicillin and ampicillin, whereas none were resistant to a cephalosporin.

CONCLUSIONS: These findings suggest that rheumatoid arthritis may be a risk factor for LPJI in older prosthetic joint patients undergoing invasive dental procedures in the posterior oral cavity. This increased risk is caused, in part, by a higher prevalence of $S$. aureus in the posterior oral cavity. The prevalence and the antibiotic resistance of $S$. aureus must be

From the "University of Michigan School of Dentistry; tUniversity of Michigan Medical School; and fUniversity of Tennessee, Memphis.

Work was completed at the University of Michigan and Veterans Administration Medical Center, Ann Arbor, Michigan.

This project was supported by NIH Grant DE07101, the VA Research Service, NIA Grant AG08808, and The Geriatric Research Center.

The paper was presented at the 71 st General Session of the International Association of Dental Research, March 1993, Chicago, Illinois.

Address correspondence to Jed J. Jacobson, DDS, MPH, School of Dentistry,

University of Michigan, 1011 N. University, Ann Arbor, MI 48109-1078. considered when determining the need for chemoprophylaxis. J Am Geriatr Soc 45:590-593, 1997.

$\mathrm{C}$ ontroversy exists regarding the management of dental patients with prosthetic joints. Late prosthetic joint infections (LPJI) can cause serious illness in older patients. ${ }^{1-4}$ Theoretically, older patients are at risk for costly, morbid LPJIs following a bacteremia associated with dental therapy. Nevertheless, because of the risk of the side effects of antibiotics, as well as the cost-effectiveness of antibiotic prophylaxis for all dental patients with prosthetic joints, chemoprophylaxis has been challenged., ${ }^{1,2}$ Rheumatoid arthritis (RA) has been suggested as a risk factor, either becuse of its demonstrated decreased granulocyte migration or the immunosuppressive agents used in its treatment. ${ }^{4-6}$ In addition, strong evidence exists that corticosteroids, used in the management of arthritic patients, alter the microbial oral flora. ${ }^{7}$

The implicated bacteria in LPJIs are mainly Staphylococcus epidermidis and Staphylococcus aurus, uncommon oral pathogens. ${ }^{8,9}$ However, prosthetic joint patients with RA and a long history of immunosuppressive therapy may have oral flora quite unlike a nonarthritic population. Specifically, $S$. epidermidis and $S$. aureus may be present more frequently in the oral cavity, which could increase the risk for LPJI. Recent evidence also suggests that $S$. aureus may be present in the subgingival flora of patients with periodontal disease. ${ }^{10}$

The purpose of this study was to determine (1) if the bacteria most often implicated in LPJIs were present in the oral cavity of an older population, and (2) if these bacteria were more prevalent in a population with RA than in an age and gender-matched nonarthritic (NA) control group. Additionally, this study sought the detection rates of $S$. aureus and S. epidermidis among several oral sites. The hypothesis that $S$. aureus and S. epidermidis are more prevalent in a rheumatoid arthritic population is based on the observation that immunosuppressive therapy has been shown to alter the oral flora.

\section{MATERIAL AND METHODS}

\section{Subjects}

The experimental population was drawn from a sample of patients treated consecutively at the Arthritis Clinic at the Veteran's Administration Medical Center and the Rheumatology Clinic at the University of Michigan Medical Center, both in Ann Arbor, Michigan. The control population was derived from the Patient Admitting and Emergency Services Clinic at the University of Michigan School of Dentistry and 
the University of Michigan Geriatric Center Human Subjects Core. Each patient provided oral and written consent.

Inclusion criteria for the experimental population included a diagnosis of rheumatoid arthritis by a rheumatologist and long-term (greater than 6 months) corticosteroid therapy. Subjects with a history of antibiotic therapy (within 6 weeks), and diabetes were excluded. The control group (NA) was selected based on age (within 2.5 years) and gender match to the experimental group. Potential control subjects were excluded if they had any major illnesses such as a history of arthritis, diabetes, antibiotic therapy (within 6 weeks) or immunosuppressive treatment. Twenty-five of 225 subjects declined participation in the study. As shown in Table 1, there were no significant differences in age or gender between the experimental (RA) and control (NA) groups.

Each subject completed a health questionnaire that investigated their history of major illnesses, prosthetic joints, persistent dry mouth, use of tobacco products, antibiotics within the recent past (within 6 weeks), and current medications. All health questionnaires were reviewed by investigators B.P. and G.A., and histories of xerostomia were clinically verified.

\section{Microbial Sampling}

Microbial samples were obtained from the following sites during the months of June, July, and August:

1. Unstimulated saliva was collected in sterile cups from 83 experimental and 83 control subjects, and $0.1 \mathrm{~mL}$ was streaked onto Colistin nalidixic acid (CNA) $5 \%$ sheep's blood agar with a sterile disposable loop. Subjects were instructed about expectoration to avoid contamination of specimen during collection. Samples were collected at least 1 hour after drinking or eating. Thirtyfour RA patients were unable to produce a salivary sample because of hyposalivation.

2. A sterile cotton-tipped applicator was placed and rotated on the posterior wall of the oropharynx of all RA and NA subjects. Thirteen experimental subjects were unable to provide a sample because of stimulation of the "gag reflex."

3. Unattached subgingival flora was obtained as described by Slots. ${ }^{11}$ Using a sterile forceps, a fine absorbent paper point was inserted to the depth of the gingival sulcus of a tooth. The paper point samples were obtained after removal of supragingival plaque with sterile gauze. Tooth selection was based on the following selection sequence: maxillary incisors, mandibular incisors, max-

\begin{tabular}{lcc}
\hline \multicolumn{3}{c}{ Table 1. Description of the Population Studied } \\
\hline & $\begin{array}{c}\text { Rheumatoid } \\
\text { Arthritis } \\
\mathrm{n}=111\end{array}$ & $\begin{array}{c}\text { Non-rheumatoid } \\
\text { Arthritis } \\
\mathrm{n}=83\end{array}$ \\
\hline Male & 74 & 50 \\
Female & 37 & 33 \\
Ratio male:female* & 2.00 & 1.52 \\
Age (years, & $58.7 \pm 11.64$ & $55.9 \pm 12.91$ \\
mean \pm S.D.) & & \\
\hline
\end{tabular}

* Chi-square, no significant difference $(P$ value $=.356)$.

* One sample student's $t$ test, no significant difference $(P$ value $=.975)$. illary molars, mandibular molars, maxillary cuspids, mandibular cuspids, and maxillary/mandibular premolars. A maximum of two subgingival sites was sampled on each subject.

Attached subgingival flora was obtained by inserting a curette to the depth of the gingival sulcus and withdrawing the instrument slowly with the cutting edge of the instrument against the root or tooth surface. The same selection sequence as described above was used. A maximum of two subgingival sites was sampled on each subject.

4. Using a sterile cotton tip applicator, samples were obtained from the posterior one-third of the tongue of 32 randomly selected RA subjects and 83 NA subjects. Before sampling, the dorsal surface of the tongue was blotted lightly with sterile gauze to remove any remaining saliva. Insufficient funds precluded sampling of all 111 RA subjects. Since previous investigators have concluded that a saliva sample is reflective of the palatel, buccal mucosa, and tongue, it was decided by the investigators to limit the tongue sampling to this randomly selected subgroup. ${ }^{12}$

All specimens were streaked on to CNA sheep's blood agar for isolation and incubated at $37^{\circ} \mathrm{C}$ for 24 hours. Staphylococcal identification was based on colony morphology, Gram-stain characteristics, catalase reaction, coagulase test, latex agglutination and API Staph Trac ${ }^{\square}$ identification.

\section{Data Analysis}

The chi-square analysis was used to assess differences in the prevalence of staphylococci among the subject groups and gender differences between experimental and control groups. A value of $P<.05$ was chosen for statistical signifcance. A student's $t$ test was employed to assess differences in mean ages of the RA and NA groups. Again, $P<.05$ was required for statistical significance.

\section{RESULTS}

A description of the study population is provided in Table 1. Although there are differences in the mean ages $(\mathrm{RA}=58.7$ years vs $\mathrm{NA}=55.9$ years $)$ and the ratio of males to females ( $\mathrm{RA}=2.00$ vs $\mathrm{NA}=1.52$ ) between the experimental and control populations, the differences are slight and not statistically significant.

The prevalence of $S$. aureus at the oral sites sampled is displayed in Table 2. Please note some subjects exhibited more than one positive site in the oral cavity. Thus, the experimental subjects grew $S$. aureus from 26 oral sites. Of

Table 2. The Prevalence of Oral Stapbylococcus aureus

\begin{tabular}{lcccc}
\hline \multicolumn{1}{c}{ Sample Site } & $\begin{array}{c}\text { Experimental } \\
(\mathrm{RA})^{*} \mathrm{n}=111\end{array}$ & $\begin{array}{c}\text { Control } \\
(\mathrm{NA}) \dagger \mathrm{n}=83\end{array}$ & $P$ Value \\
\hline Oro-pharynx & $13 / 104(12.5 \%)$ & $3 / 83(3.6 \%)$ & $P=.031$ \\
Saliva & $7 / 83$ & $(8.4 \%)$ & $3 / 83(3.6 \%)$ & $P=.192$ \\
Tongue & $4 / 32$ & $(12.5 \%)$ & $1 / 83(1.2 \%)$ & $P=.008$ \\
Gingival sulcus & $2 / 82$ & $(2.4 \%)$ & $2 / 83(2.4 \%)$ & $P=.990$ \\
Total subjects $\neq$ & $16 / 111(14.4 \%)$ & $3 / 83(3.6 \%)$ & $P=.012$ \\
\hline
\end{tabular}

* RA = Rheumatoid arthritis.

$+\mathrm{NA}=$ No arthritis.

$\ddagger$ Some subjects grew out $S$. aureus at more than one site. 
the 194 experimental and control subjects examined, only 19 subjects (16 RA and $3 \mathrm{NA}$ ) had $S$. aureus cultured from the oral sites sampled. The higher prevalence of $S$. aureus in the RA population, $14.4 \%$ (16/111), compared with the NA population, $3.6 \%(3 / 83)$, is statistically significant (Table 3 ).

Statistically significant differences $(P<.05)$ between RA and NA patients were found in the prevalence of $S$. aureus in the oropharynx $(\mathrm{RA}=13 / 104$ vs $\mathrm{NA}=3 / 83$ ) and the tongue ( $4 / 32$ vs $1 / 83)$. The remaining oral sites revealed either no growth or statistically insignificant differences in prevalence of $S$. aureus. However, it should be noted that the prevalence of $S$. aureus in the saliva of the RA population was nearly twice that of NA subjects.

Table 3 illustrates the distribution of $S$. aureus positive sites in the RA population studied. Of the 111 subjects sampled, $16(14.41 \%)$ revealed the presence of $S$. aureus in at least one of the oral sites. Thirteen of the 16 RA subjects had $S$. aureus cultured from their oropharynx. Additionally, all but one RA patient (\#36) with $S$. aureus detected in the saliva also had $S$. aureus cultured from the oropharynx. These findings suggest that the posterior oropharynx, not the gingival sulcus or tongue, may be the source of oral $S$, aureus.

Table 4 displays the association between oral $S$. aureus and xerostomia. There is strong evidence $(P$ value $<.05)$ that xerostomia and the prevalence of $S$. aureus are associated.

Staphylococcus epidermidis was not detected in any of the oral sites sampled in the study.

The results of the antibiotic sensitivity of oral $S$. aureus are seen in Table 5 . A random selection of 15 cultures of $S$. aureus from all strains of $S$. aureus collected during the study was used for this analysis. Not unexpectedly, a high rate of resistance to penicillin $(69 \%)$ and ampicillin $(73 \%)$ was encountered. Further, no isolates were resistant to cefaclor, which simply means that none of these isolates were methi-

Table 3. Distribution of $S$. aureus Positive Sites in Rheumatoid Arthritis Subjects

\section{Culture Sites}

Age

Subject (yrs) Gender Oropharynx Saliva Tongue Sulcus

$\begin{array}{lllllll}001 & 71 & M & + & 0 & 0 & 0 \\ 003 & 48 & M & + & 0 & 0 & 0 \\ 014 & 62 & M & + & + & 0 & 0 \\ 017 & 64 & M & + & + & 0 & 0 \\ 018 & 40 & M & + & 0 & 0 & 0 \\ 019 & 61 & M & + & + & 0 & 0 \\ 023 & 57 & M & + & 0 & 0 & 0 \\ 036 & 62 & F & 0 & + & 0 & 0 \\ 043 & 44 & M & + & + & 0 & 0 \\ 064 & 74 & M & + & 0 & 0 & 0 \\ 078 & 36 & M & + & 0 & 0 & 0 \\ 079 & 74 & M & + & + & 0 & + \\ 081 & 69 & M & 0 & 0 & + & + \\ 083 & 70 & F & + & 0 & + & 0 \\ 085 & 63 & M & + & + & + & 0 \\ 090 & 73 & M & 0 & 0 & + & 0\end{array}$

Average: 60.5 Totals:
Table 4. Xerostomia and Prevalence of Oral Stapbylococcus aureus in Rheumatoid Arthritis Subjects

\begin{tabular}{lcc}
\hline & Xerostomia & No Xerostomia \\
\hline (+) Culture for S. aureus & 8 & 8 \\
$(-)$ Culture for S. aureus & 15 & 80 \\
Total $(n=111)$ & 23 & 88 \\
\hline
\end{tabular}

Chi-square $P$ value $=.002$.

Table 5. The Antibiotic Sensitivity of Oral S. aureus

\begin{tabular}{lccc}
\hline & \multicolumn{3}{c}{ Zones of Inhibition } \\
\cline { 2 - 4 } \multicolumn{1}{c}{ Antibiotic } & Resistant & Intermediate & Susceptible \\
\hline Penicillin & 9 & 0 & 6 \\
Ampicillin & 9 & 0 & 6 \\
Amoxicillin with & 0 & 0 & 15 \\
$\quad$ clavulonic acid & & & \\
Erythromycin & 1 & 7 & 7 \\
Doxycycline & 0 & 0 & 15 \\
Clindamycin & 0 & 0 & 15 \\
Vancomycin & 0 & 0 & 15 \\
Gentamycin & 0 & 0 & 15 \\
Cefaclor (a & 0 & 2 & 13 \\
$\quad$ cephalosporin) & & & \\
\hline
\end{tabular}

cillin-resistant. One-half of the $S$. aureus isolates revealed intermediate or lesser zones of inhibition to erythromycin.

\section{DISCUSSION}

Before conclusions are reached, the limitations of this clinical study need to be recognized and their impact fully appreciated. Because the purpose of this study was to establish the prevalence of $S$. aureus in rheumatoid arthritis and in an age-gender matched control, we did not attempt to determine the relative quantity of $S$. aureus. Quantitative studies would be significant since previous investigations have shown a direct correlation between the level of bacteremia and subsequent LPJIs. ${ }^{13}$ Further, the incidence of $S$. aureus and $S$. epidermidis was not established in this study. One assumes that the presence of these bacteria would change, and this change might be attributed to the rheumatoid arthritis and/or the immunosuppressive agents used to control it. Six experimental patients underwent repeat sampling 6 months after the initial oral sampling. Two subjects went from positive to negative cultures of $S$. aureus, two switched from negative to positive, and two remained unchanged. In these two subjects, one does not know if this condition represents loss and reacquisition of an organism or persistence of the same organism. Although the question of oral microbial flora changes over time needs to be addressed in a larger study, one can still reach conclusions about the prevalence of these oral microbes in the present study.

One of the issues central to the controversy about management of dental patients with prosthetic joints is whether the bacteria implicated as the causative microbes of LPJI are present in the oral cavity of these patients. The findings from this study suggests that adult patients with rheumatoid ar- 
thritis have a significantly higher prevalence of oral S. aureus, particularly in the posterior areas (oropharynx and posterior tongue) than an age and gender-matched control population (Table 3). Although the RA population demonstrated a prevalence of $S$. aureus nearly four times higher than the NA control group (14.4\% vs $3.6 \%)$, the oral sites on and around teeth revealed no differences. The prevalence of $S$. aureus in the RA population compares well to reported detection rates $(\mathbf{1 8 \%})$ of Köndell in a "chronically ill and aged" population. ${ }^{14}$

The detection pattern (Table 3 ) seen in the rheumatoid arthritis population suggests that the source of the oral $S$. aureus may be the oropharynx. The internal nares are probably the main reservoir of $S$. aureus, and colonization of the mucosal surfaces proceeds into the oropharynx and other mucosal surfaces of the posterior oral cavity. These results are supported by previous reports, which conclude that $S$. aureus carriage in the nose ranges from 0 to $50 \%$ in healthy individuals whereas 41 to $70 \%$ of hospital patients were positive for $S$. aureus. ${ }^{14}$

The findings from this study can be interpreted in several ways. First, the results suggest that adult rheumatoid arthritis patients have a significantly higher prevalence of oral $S$. aureus than an age and gender-matched control population (Table 2). Second, the data support the view that $S$. aureus colonizes from the nares to the orophayn $x$ to the tongue, but less to the saliva or the gingival sulcus. Therefore, although RA patients with prosthetic joints may have a higher prevalence of $S$. aureus, the location of oral $S$. aureus is such that dental procedures involving the teeth and gingival crevice may not predispose an individual to a bacteremia with $S$. aureus. The exception would be invasive dental procedures that manipulate the posterior oral cavity or oropharynx.

Another commonly implicated etiological bacteria for LPJIs, S. epidermidis, was not detected in any of the oral sites of the subjects in this study. Consequently, older prosthetic joint patients, with or without rheumatoid arthritis, do not appear to be at a greater risk for LPJ1 from oral S. epidermidis.

Although the detection of $S$. aureus and/or S. epidermidis in a bacteremia of oral origin is rare, it is not unexpected when we consider the population used in previous studies, ${ }^{15}$ which is why this investigation was undertaken. From the results of this study, one could hypothesize that $S$. aureus would be more prevalent in an older, rheumatoid arthritis population with complaints of xerostomia than in a generally younger, healthy population that was the basis of previous bacteremic studies.

Not surprisingly, the $S$. aureus isolated from the study population had a high rate of resistance to penicillin $(69 \%)$ and ampicillin $(73 \%)$, whereas no isolates were resistant to a cephalosporin (Table 5). Additionally, erythromycin was only moderately effective in inhibiting the growth of $S$. aureus. Consequently, when determining the need for chemoprophylaxis before dental procedures, the high rate of resistance of $S$. aureus must be considered.

The question of the reasons for the higher prevalence of S. aureus in the oral cavity of older rheumatoid arthritis subjects remains. There is evidence that this higher prevalence is attributable to the disease itself, as well as to the immunnosuppression these patients may be experiencing through drug therapy. ${ }^{8} \mathrm{~A}$ common complication of rheumatoid arthritis is the sicca complex, which includes a decreased salivary flow resulting in xerostomia. Potentially this compli- cation results in a altered milieu, including decreased immunnoglobins from the saliva itself, leading to an altered oral flora. Although this study did not attempt to quantify the degree to which a decreased salivary flow affects the colonization of the oral cavity with staphylococcus, there is strong evidence (Table 4) that an association does exist between xerostomia and oral $S$. aureus. Further quantitative studies are needed to establish whether a decreased salivary flow enhances or results in oral colonization with $S$. aureus.

The results of this study show that patients with RA are more likely than controls to carry $S$. aureus. Additional studies investigating the prevalence of $S$. aureus in a bacteremia of oral origin are needed to determine if this older rheumatoid arthritis population is at an increased risk of LPJI. Presumably this increased risk would be caused by a higher prevalence of oral $S$. aureus, a bacteria often implicated as the cause of LPJI, on the oro-pharynx and posterior tongue. Any chemoprophylaxis regimen during dental treatment should take into consideration the high rate of resistance of $S$. aureus to penicillin, ampicillin, and, possibly, erythromycin. A prudent approach to all prosthetic joint patients seeking dental care should include a thorough history to establish if the patient has any risk factors for LPJI. Following this investigation, if one concludes that chemoprophylaxis is in the patients best interest, the choice of antimicrobials should be driven by the bacterial species one is attempting to diminish.

\section{ACKNOWLEDGMENTS}

The authors acknowledge Dr. Jonathan Ship for his critical review of the manuscript.

\section{REFERENCES}

1. Little JW. The need for antibiotic coverage for dental treatment of patients with joint replacements. Oral Surg Oral Med Oral Pathol 1983;55:20-23.

2. Jacobson JJ, Schweitzer SO, Kowalski CJ. Chemoprophylaxis of prosthetic joint patients during dental treatment: A decision-utility analysis. Oral Surg Oral Med Oral Pathol 1991;72:167-177.

3. Maderazo EG, Judson S, Pasuernak H. Late infections of total joint prostheses. Clin Orthop 1988;229:131-142.

4. Fitzgerald RH, Nolan DR, Ilstrup et al. Deep wound sepsis following total hip arthoplasty. J Bone Joint Surg 1977;59A:847.

5. Petty W, Bryan RS, Coventry MB et al. Infection after total knee arthroplasty. Orthop Clin North Am 1975;6:1005.

6. Roles NC. Infection in total prosthetic replacement of the hip and knee joints. Proc R Soc Med 1971;64:10.

7. Bartels HA, Cohen C, Scopp IW. Alterations in the oral microbial flora accompanying local and systemic drug therapy. J Periodontol 1969;40:421423.

8. Inman RD, Gallegos KV, Brause BD et al. Clinical and microbial features of prosthetic joint infection. Am J Med 1984;77:47-53.

9. Thyne GM, Ferguson JW. Antibiotic prophylaxis during dental treatment in patients with prosthetic joints. J Bone Joint Surg 1991;738:191-194.

10. Rams TE, Fei KD, Slots J. Staphylococci in human periodontal diseases. Oral Microbiol Immunol 1990;5;29-32.

11. Slots J, Rams TE, Listgarten MA. Yeasts, enteric rods and pseudomonas in the subgingival flora of seven adult periodontitis. Oral Microbiol Immunol $1988 ; 3: 47-52$

12. Piochi BJA, Zelante F. Contribution to the study of Staphylococcus isolated of the mouth. J. Staphylococcus isolated of the saliva. Rev Fac Odont S Paulo 1973;11:367-378.

13. Blomgren $G$, Lindgren $U$. Late hematogenous infection in total joint replacement: Studies of gentamicin and bone cement in the rabbit. Clin Orthop Rel Res 1981;155:244-248.

14. Köndell PA, Nord CE, Nordenram C. Characterization of Staphylococcus aurcus from oral surgical outpatients compared to isolates from hospitalized and non-hospitalized individuals. Int J Oral Surg 1984;13:416-422.

15. Pallasch PJ, Slots J. Antibiotic prophylaxis and the medically compromised patient. Periodontology 2000 1996;19:107-138. 